\title{
Microscopic Findings Biospecimen Usability
}

National Cancer Institute

\section{Source}

National Cancer Institute. Microscopic Findings Biospecimen Usability. NCI Thesaurus.

Code C119881.

A qualitative or quantitative measure that describes a biospecimen's suitability for microscopic testing purposes. 